\title{
THE EFFECTS OF IRREVERSIBILITY AND UNCERTAINTY ON CAPITAL ACCUMULATION
}

Andrew B. Abel

Janice C. Eberly

Working Paper 5363

\section{NATIONAL BUREAU OF ECONOMIC RESEARCH 1050 Massachusetts Avenue \\ Cambridge, MA 02138 \\ November 1995}

We thank Domenico Cuoco, Andrew Lo and seminar participants at the NBER for helpful discussions, and the National Science Foundation for financial support. This work was completed while Eberly was visiting Harvard University and the National Bureau of Economic Research; their hospitality is gratefully acknowledged, as is the support of a Sloan Foundation Research Fellowship. This paper is part of NBER's research programs in Asset Pricing, Economic Fluctuations and Monetary Economics. Any opinions expressed are those of the authors and not those of the National Bureau of Economic Research.

(C) 1995 by Andrew B. Abel and Janice C. Eberly. All rights reserved. Short sections of text, not to exceed two paragraphs, may be quoted without explicit permission provided that full credit, including $\odot$ notice, is given to the source. 


\title{
THE EFFECTS OF IRREVERSIBILITY \\ AND UNCERTAINTY ON CAPITAL ACCUMULATION
}

\begin{abstract}
When investment decisions cannot be reversed and returns to capital are uncertain, the firm faces a higher user cost of capital than if it could reverse its decisions. This higher user cost tends to reduce the firm's capital stock. Opposing this effect is the irreversibility constraint itself: when the constraint binds, the firm would like to sell capital but cannot. This effect tends to increase the firm's capital stock. We show that a firm with irreversible investment may have a higher or a lower expected capital stock, even in the long run, compared to an otherwise identical firm with reversible investment. Furthermore, an increase in uncertainty can either increase or decrease the expected long-run capital stock under irreversibility relative to that under reversibility. However, changes in the expected growth rate of demand, the interest rate, the capital share in output, and the price elasticity of demand all have unambiguous effects.
\end{abstract}

Andrew B. Abel

Department of Finance

The Wharton School

2315 Steinberg-Dietrich Hall

3620 Locust Walk

University of Pennsylvania

Philadelphia, PA 19104-6367

and NBER
Janice C. Eberly

Department of Finance

The Wharton School

2315 Steinberg-Dietrich Hall

3620 Locust Walk

University of Pennsylvania

Philadelphia, PA 19104-6367

and NBER 


\section{Introduction}

When a firm facing uncertainty chooses its capital stock knowing that its investment is irreversible, the firm faces a higher user cost of capital than if its investment were reversible. The user cost under irreversibility is higher the more uncertain are the returns to capital. Do these results necessarily imply that irreversibility and uncertainty reduce investment in the long run and therefore reduce capital accumulation? We show that the answer to this question is no, and that the implications of irreversibility and uncertainty are not so simply characterized. In fact, there is an opposing effect inherent in the irreversible investment model under uncertainty which tends to increase capital accumulation -- the more so the higher is uncertainty.

When investment is costlessly reversible as in the standard neoclassical model, the optimal investment policy maintains the marginal revenue product of capital equal to the user cost derived by Jorgenson (1963). However, when investment is irreversible, the optimal investment policy is to purchase capital only as needed to prevent the marginal revenue product of capital from rising above an optimally-derived trigger. This trigger, which is the user cost of capital appropriately defined to take account of irreversibility and uncertainty, is higher than the Jorgensonian user cost. Thus, if the firm currently has no capital and faces a given marginal revenue product schedule (as a decreasing function of the capital stock), the optimal capital stock under 
irreversibility is smaller than the optimal capital stock under reversibility. This result, which we will call the "user-cost" effect, occurs because the firm anticipates that the irreversibility constraint may bind in the future and thus is more reluctant to invest today; ${ }^{1}$ this finding has been emphasized by Bertola (1988), Pindyck (1988), Dixit (1989), Bertola and Caballero (1994), and Dixit and Pindyck (1994). A related result is that an increase in the variance of the shocks facing the firm tends to increase the user cost under irreversibility without affecting the user cost in the standard reversible case. This increase in the user cost due to increased uncertainty tends to further reduce the optimal capital stock under irreversibility.

The results described above apply to a firm that currently has zero capital, such as a new firm just getting started. But what are the effects of irreversibility and increased uncertainty for an ongoing firm? To address this question we focus on the long-run capital stock. Of course, in the presence of uncertainty the capital stock does not converge to a constant in the long run. Therefore, we focus on the current expectation of the capital stock $K_{t}$ at a future date $t$ and then examine long-run behavior by letting $t$ approach infinity.

The user-cost effect described above suggests that the introduction of irreversibility tends to reduce the current

\footnotetext{
${ }^{1}$ It is important for this result that the marginal revenue product of the firm is a decreasing function of the capital stock, as noted by Pindyck (1993) and Abel and Eberly (1993). If the marginal revenue product does not depend on the capital stock, then current and future marginal revenue products are unaffected by today's investment, so the link from today's investment to future returns is broken. The firm is then no more reluctant to invest under irreversibility than with reversible investment.
} 
expectation of $K_{t}$. However, there is an effect working in the opposite direction because the firm has a past as well as a future. The firm will arrive at any future date with a capital stock representing the cumulation of investment prior to that date (taking account of depreciation). If demand for the firm's output is extremely low at date $t$, the firm would like to sell some of its capital at a positive price. However, under irreversibility, the firm cannot sell capital, and it would be constrained by its own past investment behavior to have a capital stock that is higher than it would choose if it could start fresh at date $t$. This dissonance between the firm's actual capital stock and the level that it would choose to hold does not reflect any failure of rationality. Instead it reflects the firm's optimal response to favorable circumstances in the past. We refer to this effect as the "hangover" effect to indicate the dependence of the current capital stock on past behavior, especially behavior that later the firm would like to reverse. ${ }^{2}$ The hangover effect can lead to a higher capital stock under irreversibility than under reversibility.

The user-cost and hangover effects have opposing implications for the current expectation of the long-run capital stock. With irreversibility, the user-cost effect tends to reduce the expected capital stock whereas the hangover effect tends to increase the expected capital stock. The two effects also give opposing answers regarding the effect of increased uncertainty on the

2 The hangover effect is an example of "hysteresis" in irreversible investment discussed by Dixit (1992). Dixit emphasizes the finding that with irreversibility past events affect current investment behavior. 
expected long-run capital stock. As we have discussed, the usercost effect implies that increased uncertainty tends to reduce the expected long-run capital stock under irreversibility. However, the hangover effect implies that increased uncertainty tends to increase the expected long-run capital stock. In this paper we analyze the effects of irreversibility and uncertainty on the expected long-run capital stock taking account of the user-cost and hangover effects together.

The literature on irreversible investment under uncertainty has attempted in various ways to assess the long-run or average effects of irreversibility and uncertainty on the capital stock. Bertola (1988) and Bentolila and Bertola (1990) examine the long-run distribution of the marginal revenue product of capital and conclude that the mean of this distribution is reduced by the presence of irreversibility -- and reduced further the higher is uncertainty. ${ }^{3}$ Dixit and Pindyck (1994, p.372-373) consider a model similar to ours and focus on the effect of uncertainty on investment. Specifically, they calculate the expected change in the logarithm of the capital stock. They conclude that in this case "a larger $\sigma$ means a lower long-run average growth rate of the capital stock, and thus less investment on average" (p. 373), where $\sigma$ measures uncertainty. ${ }^{4}$

\footnotetext{
3 Bertola (1992) considers a deterministic model of employment with hiring and firing costs. He finds that with positive discounting and/or attrition, hiring costs tend to reduce average employment, while firing costs increase average employment. Obviously, he does not consider the effects of uncertainty.

${ }^{4}$ Specifically, Dixit and Pindyck calculate the expected long-run average change in the log of the capital stock.
} 
We calculate directly the expected future value of the capital stock. For a firm starting with a zero capital stock, the user-cost effect initially causes the firm to accumulate less capital, an effect that is magnified by increased uncertainty. As the firm accumulates capital, however, it becomes more likely that during a low-demand episode the firm would like to sell capital, if it could. The hangover effect is then operative, increasing the capital stock under irreversibility relative to that under reversibility. In the long run, we find cases in which the user-cost effect dominates, and others in which the hangover effect dominates. Increasing uncertainty does not resolve this ambiguity, but instead deepens it. We show that in the long run, increased uncertainty increases the expected capital stock under both reversibility and irreversibility. ${ }^{5}$ Whether the increase in the expected long-run capital stock is larger under reversibility or under irreversibility depends on the choice of parameter values.

In the next section of the paper, we construct a simple model of a firm with an infinite horizon and characterize its optimal investment decision with irreversibility and uncertainty. In Section III, we calculate the expected value of the capital stock, comparing the irreversible investment case to the reversible investment case and decomposing the user-cost and hangover effects. Section IV focuses on the effects of uncertainty on the long-run capital stock, while Section V considers the effects of

\footnotetext{
${ }^{5}$ This result holds for $M(\theta)$-preserving increases in uncertainty (a concept that is introduced in section IV) for positive values of $\theta$ less than one. In particular, it holds for the types of increases in uncertainty examined by Caballero (1991) and Pindyck (1993), which are $M(\theta)$-preserving with $\theta=1 / \varepsilon$.
} 
growth, the interest rate, capital share in production, and the price elasticity of demand. In Section VI we offer concluding remarks.

\section{The Firm's Optimization Problem}

We develop a simple model of the firm in order to focus on the key elements of our question: the effects of irreversibility and uncertainty on capital accumulation in the long run. Accordingly, we assume that investment is irreversible, returns to capital are uncertain, and the firm has an infinite horizon. The specific functional forms we use are chosen for their tractability, and are also used by Bertola (1988), Bertola and Bentolila (1990), Dixit (1991), and Abel and Eberly (1995).

Consider a firm that faces an isoelastic demand curve

$$
Q_{t}=X_{t} P_{t}^{-\varepsilon}
$$

where $Q_{t}$ is the quantity of output demanded, $P_{t}$ is the price of output, $X_{t}$ is a stochastic demand shock, and $\varepsilon>1$ is the price elasticity of demand. The firm produces nonstorable output $Q_{t}$ according to the Cobb-Douglas production function

$$
Q_{t}=L_{t}{ }^{1-\beta_{K}} \beta
$$

where $L_{t}$ is labor, $K_{t}$ is the capital stock, and the capital share $\beta$ satisfies $0<\beta<1$. At each point of time the firm chooses $L_{t}$ to maximize its operating profit $P_{t} Q_{t}-w L_{t}$ where $w$ is the wage rate, 
which is assumed constant. The maximized value of operating profit is given by

$$
\pi\left(K_{t}, X_{t}\right)=\frac{h}{1-\gamma} X_{t}^{\gamma} K_{t}^{1-\gamma}
$$

where $\frac{1}{\varepsilon}<\gamma \equiv \frac{1}{1+\beta(\varepsilon-1)}<1$ and $h \equiv(1-\gamma)\left(\frac{1}{\gamma \varepsilon}\right)^{\gamma / \varepsilon}(\gamma \varepsilon-1)^{\gamma-1} w^{1-\gamma \varepsilon}$.

Because the instantaneous operating profit of the firm depends on the firm's capital stock $K_{t}$ and on the stochastic component of demand $X_{t}$ the evolution of the operating profit depends on the evolution of $X_{t}$ and $K_{t}$ over time. Assume that the demand shock $X_{t}$ evolves exogenously according to a geometric Brownian motion

$$
\frac{d X_{t}}{X_{t}}=\mu d t+\sigma d z, \quad \sigma>0
$$

where $X_{0}>0$, and $d z$ is an increment to a standard Wiener process, with $E\{d z\}=0$ and $(d z)^{2}=d t$.

The firm can purchase capital at a constant price $b>0$ but is unable to sell capital. The capital stock does not depreciate. ${ }^{6}$ Therefore, the evolution of the capital stock $K_{t}$ depends only on the firm's purchases of capital. ${ }^{7}$

\footnotetext{
${ }^{6}$ This assumption greatly simplifies the calculation of the expected future capital stock. Specifically, for any time $t$ at which the firm does not purchase capital, the capital stock equals the capital stock at date $s$, where $s$ is the latest time prior to $t$ that the firm purchased capital. If there were depreciation, then the capital stock at time $t$ would have to be adjusted for depreciation since time $s$, and this adjustment would require information about the length of time $(t-s)$ since the firm's most recent purchase of capital.

7 Because the cost of adjustment is linear, the rate of investment (i.e., investment per unit of time) can be infinite. The capital stock therefore follows a continuous, but nondifferentiable (with respect to time), path.
} 
Assume that the firm is risk-neutral and discounts future cash flows at the constant rate $r>0$, where $r>\mu$. ${ }^{8}$ The investment policy that maximizes the value of the firm is derived in Appendix

A. This policy is easily expressed in terms of the marginal revenue product of capital and the user cost of capital, which we present below. The marginal revenue product of capital is $h y^{\gamma}$ where $y \equiv X / K$. The user cost of capital is

$$
c \equiv\left(1-\frac{\gamma}{\alpha_{N}}\right) r b
$$

where $\alpha_{N}$ is the negative root of the quadratic equation

$$
\rho(\eta) \equiv-\frac{1}{2} \sigma^{2} \eta^{2}-\left(\mu-\frac{1}{2} \sigma^{2}\right) \eta+r=0 .
$$

The user cost of capital in equation (5a) is the natural extension of the Jorgensonian user cost of capital to the case of irreversibility under uncertainty. More precisely, it can be shown (see Abel and Eberly (1995)) that $c$ is the sum of: (1) the interest $\operatorname{cost} r b$; and (2) the expected capital loss on a marginal unit of capital. ${ }^{9}$

The optimal investment policy is a "barrier control" policy according to which the firm purchases capital as necessary to prevent the marginal revenue product of capital from rising above the user cost $c$. When the marginal revenue product of capital is lower than $c$, it is optimal not to purchase capital. Only when the marginal revenue product of capital equals the user cost is it

8 The expected present value of operating profits, $E_{1}\left\{\int_{0}^{\infty} e^{-r s} \pi\left(K_{t+s}, X_{t+s}\right) d s\right\}$, is finite if $\mu<r$. Appendix A provides a rigorous treatment of this issue. ${ }^{9}$ More generally, the user cost contains a term reflecting the physical depreciation of capital, but in this model we have assumed that capital does not depreciate. 
optimal to purchase capital. Under this barrier control policy the marginal revenue product of capital, $h y^{\gamma}$, is a regulated geometric Brownian motion, where $h y^{\gamma} \leq c$, or equivalently $y \leq(c / h)^{1 / \gamma} \equiv y_{U}$ To describe its behavior more formally, we first characterize the behavior of $y^{\theta}$ where $\theta$ is an arbitrary positive constant. Using Ito's Lemma and recalling that $y \equiv X / K$, the behavior of $y^{\theta}$ for $y$ $<y_{U}$ is given by

$$
d y^{\theta}=M(\theta) y^{\theta} d t+S(\theta) y^{\theta} d z
$$

where $M(\theta) \equiv \frac{1}{d t} E\left\{\frac{d X^{\theta}}{X^{\theta}}\right\}=\theta \mu+\frac{1}{2} \theta(\theta-1) \sigma^{2}$

and

$$
S(\theta) \equiv \theta \sigma
$$

Thus, $M(\theta)$ is the expected rate of drift in $y^{\theta}$, and $S(\theta)$ is the instantaneous standard deviation of $y^{\theta}$. The behavior of the marginal revenue product of capital, more precisely, $d\left(h y^{\gamma}\right) /\left(h y^{\gamma}\right)$, is given by equations (6a,b,c) by setting $\theta=\gamma$. Bertola (1988) shows that the marginal revenue product of capital will have a nondegenerate ergodic distribution if and only if $M(\gamma)>$ $(1 / 2)[S(\gamma)]^{2}$. Using equations $(6 \mathrm{~b}, \mathrm{c})$ this condition can be written as

$$
\mu>\frac{1}{2} \sigma^{2}
$$

For the remainder of this paper we restrict attention to cases in which the marginal revenue product of capital has a nondegenerate ergodic distribution and therefore we assume that equation (7) holds. 


\section{The Expected Value of the Capital Stock}

The optimal investment policy of the firm is to purchase capital whenever it is needed to keep the marginal revenue product of capital, $h y^{y}$, from rising about the user cost $c$.

Recalling that $y \equiv X / K$, the marginal revenue product of capital is $h(X / K)^{\gamma}$, and the condition that the marginal revenue product of capital is always less than or equal to $c$ implies

$$
X_{t} \leq(c / h)^{1 / \gamma_{K}}
$$

Because we have assumed that capital does not depreciate, we have

$$
K_{t}=(c / h)^{-1 / \gamma} \max _{s \leq t} X_{s}
$$

Suppose that the firm is born at time 0 (without any initial capital) and normalize the demand process $X_{t}$ such that $X_{0}=1$. In this case, the expected value, as of date 0 , of the capital stock at any date $t \geq 0$ is

$$
E_{0}\left\{K_{t}\right\}=(c / h)^{-1 / \gamma} E\left\{\max _{0 \leq s \leq t} X_{s} \mid X_{0}=1\right\}
$$

The expected value of the maximum in equation (10) is ${ }^{10}$

\footnotetext{
${ }^{10}$ The expected value of $\max _{0 \leq s \leq t} X_{s}$ given $X_{0}=1$ can be calculated by defining $W \equiv \ln X$ and observing that $\max _{0 \leq s \leq} X_{s}=\exp \left(\max _{0 \leq s \leq t} W_{s}\right)$. The distribution of the maximum of $W_{s}$ (an arithmetic Brownian motion) is taken from Harrison (1985), p 13, equation (8):

$F_{l}(w) \equiv \operatorname{Pr}\left\{\max _{0 \leq s \leq t} W_{s} \leq w W_{0}=0\right\}=\Phi\left(\frac{w-\mu_{W} t}{\sigma_{w} t^{1 / 2}}\right)-e^{2 \mu_{w} w \sigma_{w}^{2}} \Phi\left(\frac{-w-\mu_{W} t}{\sigma_{w} t^{1 / 2}}\right)$. The desired expectation is $E\left\{\max _{0 \leq s \leq} X_{s} \mid X_{0}=1\right\}=\int_{0}^{\infty} e^{n} F_{t}^{\prime}(w) d w$. Tedious calculation shows that this equation is equivalent to equation (11) in the text. Alternatively, the
} 


$$
\begin{aligned}
E\left\{\max _{0 \leq s \leq t} X_{s} \mid X_{0}=1\right\}= & \frac{\mu+\frac{1}{2} \sigma^{2}}{\mu} \Phi\left(\frac{\mu+\frac{1}{2} \sigma^{2}}{\sigma} t^{1 / 2}\right) \exp [\mu r t] \\
& +\frac{\mu-\frac{1}{2} \sigma^{2}}{\mu} \Phi\left(-\frac{\mu-\frac{1}{2} \sigma^{2}}{\sigma} t^{1 / 2}\right)
\end{aligned}
$$

where $\Phi(\cdot)$ is the standard normal c.d.f. The expected value of the capital stock at any date $t \geq 0$ can be calculated by substituting equation (11) into equation (10) to obtain

$$
\begin{gathered}
E_{0}\left\{K_{t}\right\}=(c / h)^{-1 / \gamma}\left[\frac{\mu+\frac{1}{2} \sigma^{2}}{\mu} \Phi\left(\frac{\mu+\frac{1}{2} \sigma^{2}}{\sigma} t^{1 / 2}\right) \exp [\mu t]\right. \\
\left.+\frac{\mu-\frac{1}{2} \sigma^{2}}{\mu} \Phi\left(-\frac{\mu-\frac{1}{2} \sigma^{2}}{\sigma} t^{1 / 2}\right)\right]
\end{gathered}
$$

In order to focus on the effect of irreversibility, we introduce the case of costlessly reversible investment for comparison. Introducing costless reversibility also simplifies notation because we can express expected future capital stocks under irreversibility relative to the corresponding expected values under the benchmark of reversibility. In the standard case of costlessly reversible investment analyzed by Jorgenson (1963), the firm continuously adjusts its capital stock to maintain the marginal

desired expectation can be taken from Goldman, Sosin, and Gatto (1979) where, using their notation, $S(t)$ is a geometric Brownian motion and $E\left\{\max _{0 \leq \delta \leq s} S(\delta) \mid S(0)=1\right\}=e^{n} V_{\max }[1,1, t]$. Setting $S(\tau)=M(\tau)=1$, and setting $r$ equal to $\mu$ in their equation (10) on p. 1116 yields our equation (11) in the text. 
revenue product of capital equal to the Jorgensonian user cost of capital. In the absence of depreciation, and with a constant purchase price of capital, the Jorgensonian user cost, $c_{j}$ equals $r b$. This equality of marginal revenue product and user cost implies that $X=\left(c_{J} / h\right)^{1 / \gamma} K^{R}$ always, where the superscript " $R$ " indicates the case of costless reversibility. Therefore,

$$
E_{0}\left\{K_{t}^{R}\right\}=\left(c_{J} / h\right)^{-1 / y} E\left\{X_{t} \mid X_{0}=1\right\}
$$

Under the geometric Brownian motion in equation (4), the expected growth rate of $X_{t}$ is $\mu$ which implies that $E\left\{X_{t} \mid X_{0}=1\right\}=e^{\mu t}$. Therefore, the expected capital stock under reversibility in equation (13) can be written as

$$
E_{0}\left\{K_{t}^{R}\right\}=\left(c_{J} / h\right)^{-1 / \gamma} e^{\mu t}
$$

Now define $\kappa(t)$ as the ratio of the expected value of the capital stock at date $t$ under irreversibility to the expected value of the capital stock at date $t$ under costless reversibility. Using this definition along with equations (10) and (13) we have

$$
\kappa(t) \equiv \frac{E\left\{K_{\imath} \mid X_{0}=1\right\}}{E\left\{K_{\imath}^{R} \mid X_{0}=1\right\}}=C \times H(t)
$$

where

$$
C \equiv\left(\frac{c}{c_{J}}\right)^{-1 / \gamma}=\left(1-\frac{\gamma}{\alpha_{N}}\right)^{-1 / \gamma}<1
$$

and

$$
H(t) \equiv \frac{E\left\{\max _{0 \leq s \leq t} X, X_{0}=1\right\}}{E\left\{X_{t} \mid X_{0}=1\right\}} \geq 1
$$


The inequality in equation (15c) holds strictly for $t>0$.

Equations $(15 a, b, c)$ illustrate the two opposing effects of irreversibility on the expected capital stock: the user-cost effect measured by $C$ and the hangover effect measured by $H(t)$. The introduction of irreversibility increases the user cost of capital relative to the user cost in the standard case of costless reversibility. This increase in the user cost tends to reduce the optimal capital stock -- as reflected in the value of $C$ less than one. Working in the opposite direction is the hangover effect. Under irreversibility, the capital stock at any date $t, K_{t}$, is proportional to $\max _{0 \leq s \leq t} X$, whereas under reversibility the capital stock at any date $t$ is proportional to the contemporaneous value of the demand shock $X_{t}$. The historical peak of $X$ is at least as large as the contemporaneous value of $X$, and this consideration, which is reflected in a value of $H(t)$ greater than (or equal to) one, tends to increase the expected value of the optimal capital stock under irreversibility. To see which effect--the user-cost effect $C$ or the hangover effect $H(t)$--is dominant, we need to compute the effect of irreversibility on the product $C \times H(t)$.

First we study the properties of $C$ and $H(t)$ separately.

Proposition 1: $0.367879=e^{-1}<e^{1 / \alpha_{N}}<C<1$.

Proof: See Appendix C.

Proposition 2: $H(0)=1$ and $1<H(\infty)=1+\frac{\sigma^{2}}{2 \mu}<2$.

Proof: Inspect equations (11) and (15c) using the facts that $E\left\{X \mid X_{0}=1\right\}=\exp (\mu t), \Phi(\infty)=1$, and $\Phi(-\infty)=0$, and the 
assumption that $\mu>(1 / 2) \sigma^{2}$.

q.e.d.

Notice that the ratio of the expected capital stocks at time 0 , $\kappa(0)$, equals $C \times H(0)=C<1$. Therefore irreversibility reduces the expected value of the initial capital stock because only the user-cost effect is operative for the initial capital stock; the hangover effect is inoperative because the firm has not yet accumulated any capital in the past. However, as time proceeds, the hangover effect becomes operative. Depending on the parameters of the problem, the expected capital stock under irreversibility may eventually exceed the expected capital stock under reversibility, or it may turn out that even in the long run the expected capital stock is lower under irreversibility than under reversibility. We illustrate various possibilities later in Figures 1 and 2 .

\section{The Effects of Increased Uncertainty}

In this section we examine the effects on the expected capital stock of an increase in uncertainty. In analyzing these effects we would like to focus on mean-preserving increases in uncertainty, but we must decide which variable has its mean preserved. The answer to this question is fairly obvious for a competitive firm facing uncertain demand, because such a firm is a price-taker. The natural--indeed only--demand variable to focus on is the price facing the firm, and thus investigations of the effect of uncertainty 
in a competitive framework typically focus on mean-preserving increases in the variance of price. ${ }^{11}$

In the current model, the firm faces the downward-sloping demand curve in equation (1), and two natural choices emerge as candidates for a mean-preserving spread. It is evident from equation (1) that if we interpret demand shocks as changes in the quantity demanded at any given price, then the relevant shock is $X_{t}$; in this case, we will study the effects of increases in uncertainty that leave the expected value of $X_{t}$ unchanged. Alternatively, the demand curve in equation (1) can be rewritten as $P_{t}=X_{t}^{1 / \varepsilon} Q_{t}^{-1 / \varepsilon}$. This formulation of the demand curve indicates that if we interpret demand shocks as changes in the price associated with any given quantity of output demanded, then the relevant shock is $X_{t}^{1 / \varepsilon}$; in this case, we will study the effects of increases in uncertainty that leave the expected value of $X_{t}^{1 / \varepsilon}$ unchanged. To accommodate both of these cases in a more general framework, we will examine changes that leave the expected value of $X_{t}^{\theta}$ unchanged, recognizing that $\theta=1$ and $\theta=$ $1 / \varepsilon$ represent the two natural cases discussed here. ${ }^{12}$

Observe that $E\left\{X_{t} \mid X_{t}=1\right\}=\exp [M(\theta) t]$ so that changes in $\mu$ and $\sigma$ that leave $M(\theta)$ unchanged also leave $E\left\{X_{t}{ }^{\theta} \mid X_{t}=1\right\}$ unchanged. Setting $M(\theta)$ equal to a constant, $M_{0}$, in equation (6b) and applying the implicit function theorem yields

\footnotetext{
11 See, for example, Hartman (1972) and Abel (1983).

12 As we see below this choice is not just a normalization; it has both qualitative and quantitative importance for the results. In their analyses of investment under uncertainty, Caballero (1991) and Pindyci (1993) adopt the second formulation discussed above, i.e., $\theta=1 / \varepsilon$.
} 


$$
\left.\frac{d \mu}{d \sigma^{2}}\right|_{M(\theta)=M_{0}}=\frac{1}{2}(1-\theta)
$$

When $\theta=1$, the expression on the right hand side of equation (16) equals zero, which means that an increase in $\sigma$ holding $\mu$ fixed is a mean-preserving spread on $X$. However, when $\theta=1 / \varepsilon<1$, the right hand side of equation (16) is positive so that an increase in $\sigma$ must be accompanied by an increase in $\mu$ in order to leave the mean of $X^{1 / \varepsilon}$ unchanged.

In Appendix B we calculate the response of the root $\alpha_{N}$ to a $M(\theta)$-preserving increase in $\sigma^{2}$, and we show that

$$
\left.\frac{d \alpha_{N}}{d \sigma^{2}}\right|_{M(\theta)=M_{0}}>0 \quad \text { if } \theta \geq 0 .
$$

Using this result, we derive the effect of $\sigma^{2}$ on $C$ in Proposition 3:

Proposition 3: If $\theta \geq 0$, then $\left.\frac{d C}{d \sigma^{2}}\right|_{M(\theta)=M_{0}}<0$.

Proof: Observe from the definition of $C$ in equation (15b) that $C$ is a decreasing function of $\alpha_{N 2}$ According to equation (17), a $M(\theta)$-preserving increase in $\sigma^{2}$ increases $\alpha_{N}$ which in turn decreases $C$.

q.e.d.

According to Proposition 3, a $M(\theta)$-preserving increase in $\sigma^{2}$ reduces $C$ and hence tends to reduce the expected capital stock under irreversibility relative to the expected capital stock under reversibility. This occurs because an increase in $\sigma^{2}$ increases the user cost of capital under irreversibility but has no effect on the user cost with reversibility. Recall, however, that $C$ captures only the user-cost effect. We must also take into account the effect on the hangover factor $H(t)$. For simplicity we focus on $H(\infty)$. 
Proposition 4: If $\theta \geq 0$, then

$\left.\frac{d H(\infty)}{d \sigma^{2}}\right|_{M(\theta)=M_{0}}=\frac{1}{2 \mu}\left[1-\frac{\sigma^{2}}{2 \mu}+\theta \frac{\sigma^{2}}{2 \mu}\right]>0$.

Proof: Differentiate the expression for $H(\infty)$ in Proposition 2 with respect to $\sigma^{2}$ using equation (16). The sign of the resulting derivative is obtained by applying the inequality in equation (7).

q.e.d.

Recall that $\kappa(\infty)=C \times H(\infty)$. We have shown that $C<1$ under uncertainty and that increases in the instantaneous variance $\sigma^{2}$ decrease $C$. Working in the opposite direction, however, are the facts that $H(\infty)>1$ under uncertainty and that $H(\infty)$ is an increasing function of the instantaneous variance $\sigma^{2}$. Whether $\kappa(\infty)$ is less than or greater than one, and whether $k(\infty)$ is an increasing or decreasing function of $\sigma^{2}$ depends on whether the user-cost effect operating through $C$ or the hangover effect operating through $H(\infty)$ is dominant. Figures 1 and 2 illustrate that in some cases the user-cost effect is dominant while in other cases the hangover effect is dominant.

Both figures present results for an example in which the interest rate, $r$, equals 0.05 , the expected growth rate of demand, $\mu$, is 0.029 , the capital share in the Cobb-Douglas production function, $\beta$, equals 0.33 , and the elasticity of demand, $\varepsilon$, equals 10. Together the values of $\beta$ and $\varepsilon$ imply that $\gamma=0.251889$.

These values are not chosen necessarily for their realism, but to 
illustrate that for admissible parameter values, a wide variety of results is possible.

In Figure 1, we examine changes in the instantaneous standard deviation $\sigma$ that leave the mean of $X$ unchanged. Recall that holding the mean of $X$ fixed corresponds to a mean-preserving spread on the shocks to the quantity of output demanded at any given price. Panel a of Figure 1 shows that as $\sigma$ increases, the user-cost factor $C$ decreases and the hangover factor $H(\infty)$ increases, as stated by Propositions 3 and 4 respectively. The behavior of $\kappa(\infty)$ is not monotonic, however, as shown in panel $b$. Also note in panel $b$ that, depending on the value of $\sigma, \kappa(\infty)$ may be greater than, less than, or equal to 1 .

\section{Figure 1:}

\section{The Long-run Effect of Uncertainty on $x(\theta=1)$}

a. The user-cost and hangover effects

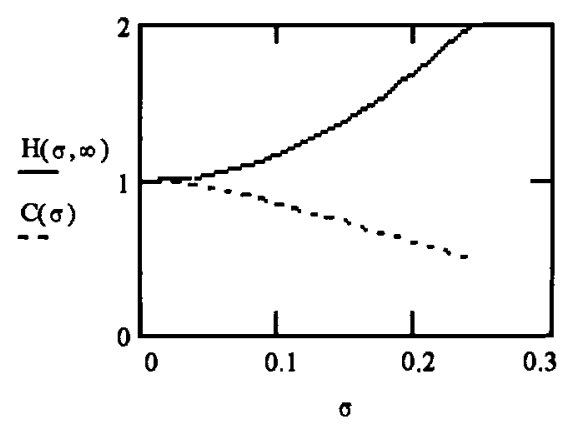


b. The total effect of uncertainty on $\kappa$

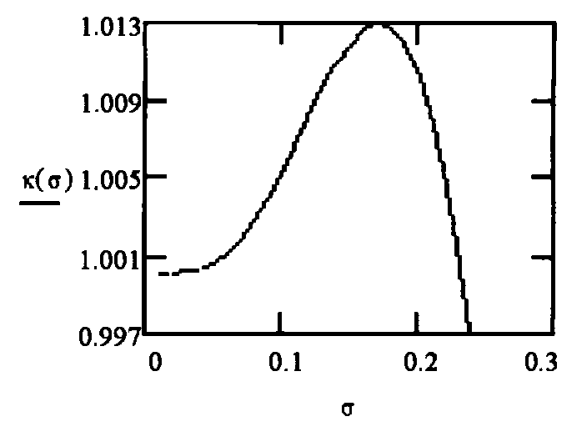

Figure 2 presents results for the case in which the mean of $X^{1 / \varepsilon}$ is held fixed, which corresponds to a mean-preserving spread on shocks to the price at any given quantity of output demanded. In this case, $\mu=0.029$ when $\sigma=0.01$, but as $\sigma$ increases, $\mu$ increases in order to keep $M(\theta)$ constant. As in Figure 1.b, $\kappa(\infty)$ is not monotonic in the instantaneous standard deviation $\sigma$, and $k(\infty)$ may be greater than, equal to, or less than one.

\section{Figure 2:}

The Long-run Effect of Uncertainty on $\kappa(\theta=1 / \varepsilon)$

a. The user-cost and hangover effects

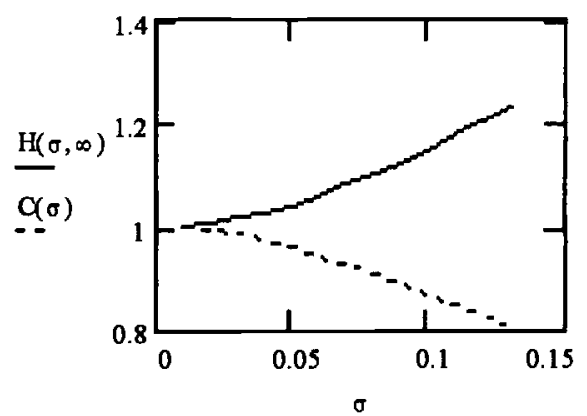


b. The total effect of uncertainty on $\kappa$

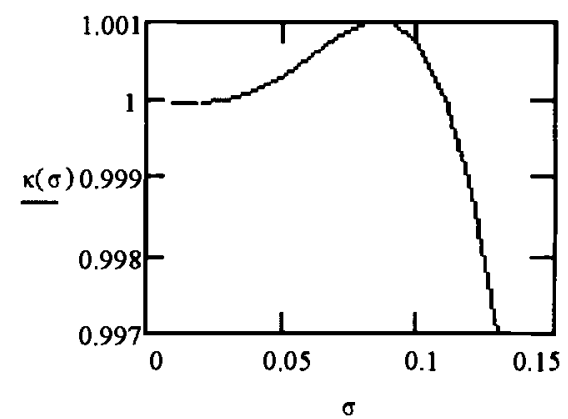

In both of these figures, $\kappa(\infty)$ does not differ greatly from one, though it can be greater or less than one depending on the value of the standard deviation, $\sigma$. For other parameter values, however, $\kappa$ $(\infty)$ can be much greater or less than one. For example, if the interest rate is $0.10, \kappa(\infty)$ in Figure $1 \mathrm{~b}$ is 1.21 when $\sigma=0.24$ and $k(\infty)$ in Figure $2 b$ is 1.04 when $\sigma=0.18$. If the expected growth rate of demand, $\mu$, is then decreased by 0.005 , with $\theta=1$ as in Figure $\mathrm{lb}, \kappa(\infty)$ is 1.24 when $\sigma=0.21$; however, with $\theta=1$ / $\varepsilon$ as in Figure $2 b, \kappa(\infty)$ falls below 0.55 as $\sigma$ reaches its maximum. ${ }^{13}$

Figures 1 and 2 and the above calculations suggest that there is considerable ambiguity both in the magnitude and the sign of the long-run effects of an increase in uncertainty. However, this analysis focuses on $\kappa(t)$, the ratio of the expected capital stock under irreversibility to the expected capital stock under reversibility. In the case of a $M(\theta)$-preserving spread with $0<\theta<$

13 The maximum allowable value of $\sigma$ comes from the restriction in equation (7) that $\mu>(1 / 2) \sigma^{2}$. In this calculation $\mu=0.024$ when $\sigma=0.01$, and $\mu$ increases as $\sigma$ increases in order to maintain $M(\theta)$ fixed. 
1, we can derive unambiguous results about the long-run effect of uncertainty on the levels of the expected capital stock under irreversibility and under reversibility. Specifically, when $0<\theta<$ 1, a $M(\theta)$-preserving increase in $\sigma^{2}$ also increases $\mu$, the drift in $X$. Eventually, the increase in the drift dominates any other effects and increases the expected capital stock. This result is based on the following lemma.

Lemma 1: Let $\chi_{j}(t) \equiv a_{j}(t) e^{\mu f}+b_{j}(t)$ where $a_{j}(t)>0$ and $b_{j}(t)$ are finite for all $t$, and $j=1,2$. If $\mu_{1}>\mu_{2} \geq 0$, then for sufficiently large $t, \chi_{1}(t)>\chi_{2}(t)$.

Proof: $\chi_{1}(t)-\chi_{2}(t)=a_{1}(t) e^{\mu_{1} t}-a_{2}(t) e^{\mu_{2} t}+b_{1}(t)-b_{2}(t)=$ $e^{\mu_{1} t}\left[a_{1}(t)-a_{2}(t) e^{\left(\mu_{2}-\mu_{1}\right) t}+\left(b_{1}(t)-b_{2}(t)\right) e^{-\mu_{1} t}\right]$. For sufficiently large $t$, the term in square brackets is positive. $\quad$ q.e.d.

Proposition 5: If $0<\theta<1$, then $\left.\frac{d E_{0}\left\{K_{t}^{R}\right\}}{d \sigma^{2}}\right|_{M(\theta)=M_{0}}>0$ and, for sufficiently large $t,\left.\frac{d E_{0}\left\{K_{t}\right\}}{d \sigma^{2}}\right|_{M(\theta)=M_{0}}>0$.

Proof: Observe from equation (16) that a $M(\theta)$-preserving spread increases $\mu$. The effect on the expected capital stock in the reversible case then follows directly from equation (14), and the effect on the expected capital stock in the irreversible case follows applying Lemma 1 to equation (12).

q.e.d.

Proposition 5 applies to a mean-preserving spread in the distribution of shocks to the price associated with any given 
quantity of output demanded $(\theta=1 / \varepsilon)$, but it does not apply to a mean-preserving spread in the distribution of shocks to the quantity of output demanded at any given price $(\theta=1)$. For the cases covered by Proposition 5, an increase in uncertainty unambiguously increases the expected long-run capital stock under irreversibility. This result is not due to irreversibility alone, however, since the expected capital stock increases under reversibility as well. Because an increase in uncertainty can either increase or decrease $\kappa(\infty)$, we cannot determine in general whether the expected long-run capital stock increases by more under irreversibility or under reversibility.

\section{The Effects of Demand Growth, the Interest Rate, the} Capital Share, and the Price Elasticity of Demand

We have seen that the expected long-run capital stock under irreversibility can be larger or smaller than under reversibility. Also, an increase in uncertainty can either increase or decrease the expected long-run capital stock under irreversibility relative to its value under reversibility. In contrast to these findings of ambiguity, in this section find that other parameters unambiguously move the expected long-run capital stock under irreversibility relative to its value under reversibility. Specifically, we show that a higher expected growth rate of demand, a higher capital share, and a higher price elasticity of demand all tend to reduce the expected long-run capital stock under irreversibility 
relative to that under reversibility, while a higher interest rate has the opposite effect.

The expected growth rate of the demand shock, $X_{t}$, is given by the drift parameter $\mu$ in equation (4). We first show that an increase in the expected growth rate of demand increases the expected capital stock under irreversibility and under reversibility. However, this effect is weaker under irreversibility, so that an increase in the expected growth rate of demand decreases the ratio of the long-run expected capital stock with irreversible investment to that under reversible investment.

Proposition 6: $\frac{d E_{0}\left\{K_{t}^{R}\right\}}{d \mu}>0$ and, for sufficiently large $t$, $\frac{d E_{0}\left\{K_{t}\right\}}{d \mu}>0$.

Proof: Inspect equation (14) and apply Lemma 1 to equation (12).

\section{q.e.d.}

According to Proposition 6, an increase in the expected growth rate of demand increases the expected long-run capital stock whether investment is reversible or irreversible. To see whether the effect is larger under reversibility or irreversibility, we must examine the effect of expected growth on the user-cost effect $C$ and on the hangover effect $H(t)$.

Proposition 7: $\frac{d C}{d \mu}=\frac{C}{\left(\gamma-\alpha_{N}\right) \rho^{\prime}\left(\alpha_{N}\right)}>0$ and $\frac{d H(\infty)}{d \mu}=-\frac{\sigma^{2}}{2 \mu^{2}}<0$. 
Proof: See Appendix D.

Notice that an increase in the growth rate of demand tends to reduce the strength of both the user-cost effect and the hangover effect, that is, both $C$ and $H(\infty)$ become closer to unity as $\mu$ rises. In this case, however, the sign of the net effect can be determined, as shown in the following corollary.

Corollary to Proposition 7: $\frac{d \kappa(\infty)}{d \mu}<0$.

Proof: See Appendix D.

A higher expected growth rate of demand therefore increases the expected long-run capital stock by more in the case of reversibility than in the case of irreversibility. This means that in a firm with high expected demand growth, we are more likely to find that irreversibility reduces its capital stock in the long run than in a firm with low expected demand growth. This result may seem surprising since the higher is demand growth, the more similar are the user costs calculated in the Jorgensonian and irreversible investment cases. ${ }^{14}$ That is, the rules governing investment become more alike as $\mu$ rises, so it may seem that the irreversibility constraint should become less important in a high growth environment. This demonstrates the importance of the

${ }^{14}$ From Appendix B, equation (B.3), an increase in $\mu$ reduces the value of the root, $\alpha_{N}<0$, which from the definition of the user cost in equation ( $5 a$ ), reduces the user cost in the irreversible case, driving it closer to $r b$, the Jorgensonian user cost. 
hangover effect, though, since this effect is weakened even more than the user-cost effect as $\mu$ rises. Thus, even while the irreversible investment rule becomes more like the reversible rule when growth is high, the expected irreversible capital stock is nonetheless driven down compared to the expected reversible capital stock.

The effect of the interest rate on the long-run capital stock is determined solely by its effect on the user-cost effect, since the hangover effect is independent of the interest rate (at any horizon). Thus, we obtain the effect of the interest rate on the relative expected capital stocks directly from the following proposition.

Proposition 8:

$\frac{d \kappa(t)}{d r}=\frac{d C}{d r} H(t)=\frac{d C}{d \alpha_{N}} \frac{d \alpha_{N}}{d r} H(t)=\frac{-\kappa(t)}{\alpha_{N}\left(\gamma-\alpha_{N}\right) \rho^{\prime}\left(\alpha_{N}\right)}>0$.

Proof: See Appendix D.

This proposition means that a higher interest rate tends to increase the long-run expected capital stock under irreversibility relative to that under reversibility by weakening the user-cost effect. Note that while the user cost under both irreversibility and reversibility rises with the interest rate -- which tends to reduce the long-run capital stock in both cases -- this effect is weaker under irreversibility than under reversibility. ${ }^{15}$ Since firms

\footnotetext{
15 From the definition of the Jorgensonian user cost in Section II, the elasticity of $c_{J}$ with respect to $r$ is one. Differentiating equation (5a) and using equation (B.4), the corresponding elasticity in the irreversible case is given by
} 
discount the costs associated with the inability to sell capital in the future, greater discounting tends to reduce the strength of the user-cost effect. This finding is consistent with the "discounting effect" emphasized by Bertola (1992) in a model of employment under certainty.

Together, the capital share, $\beta$, and the price elasticity of demand, $\varepsilon$, determine the concavity of the profit function, measured by the coefficient $\gamma$. Here we examine the effect of changing $\gamma$ on the expected long-run capital stock under irreversibility compared to reversibility. Notice that the hangover effect, $H(t)$, in equation (15c) is independent of $\gamma$, so we need only examine the user-cost effect, $C$, to determine the effect of $\gamma$ on the expected capital stock at any horizon.

\section{Proposition 9:}

$$
\frac{d \kappa(t)}{d \gamma}=\frac{d C}{d \gamma} H(t)=\frac{\kappa(t)}{\gamma^{2}}\left[\ln \left(1-\frac{\gamma}{\alpha_{N}}\right)-\frac{\gamma}{\gamma-\alpha_{N}}\right]>0 \text {. }
$$

Proof: See Appendix D.

Thus, an increase in $\gamma$ tends to increase the capital stock under irreversibility relative to that under reversibility. This occurs because as $\gamma$ rises, the profit function becomes more concave $\frac{d c}{d r} \frac{r}{c}=1-\frac{r \gamma}{\left(1-\frac{\gamma}{\alpha_{N}}\right) \alpha_{N}{ }^{2} \rho^{\prime}\left(\alpha_{N}\right)}=1-\frac{r \gamma}{\left(\alpha_{N}-\gamma\right) \alpha_{N} \rho^{\prime}\left(\alpha_{N}\right)}$. Using equation (B.1) and the fact that $\alpha_{N} \rho^{\prime}\left(\alpha_{N}\right)=\rho\left(\alpha_{N}\right)-\left(r+\frac{1}{2} \sigma^{2} \alpha_{N}^{2}\right)=-\left(r+\frac{1}{2} \sigma^{2} \alpha_{N}^{2}\right)$, this can be simplified to $\frac{d c}{d r} \frac{r}{c}=1-\frac{r}{r+\frac{1}{2} \sigma^{2} \alpha_{N}^{2}} \frac{\gamma}{\gamma-\alpha_{N}}$, which implies $0<\frac{\partial c}{\partial r} \frac{r}{c}<1$. Thus while in both cases the user cost increases with the interest rate, the elasticity in the irreversible case is less than that in the reversible case. 
(observe that with $\gamma=0$ the profit function is linear in $K$ ) and thus deviations from the optimal reversible capital stock are more costly to the firm. ${ }^{16}$ Thus the value of $C$ increases toward 1 and the expected capital stock under irreversibility increases relative to that under reversibility. Notice from the definition of $\gamma$ in equation (3) that $\gamma$ is negatively related to the capital share $\beta$ and the price elasticity of demand $\varepsilon$. Thus, a reduction in either the elasticity of demand or the capital share will be associated with an increase in the expected capital stock under irreversibility relative to the expected capital stock under reversibility. This result occurs because with relatively inelastic demand or a low capital share, it is more costly for the firm to deviate from the optimal reversible capital stock, so the user-cost effect $C$ increases toward one.

\section{Concluding Remarks}

When investment is irreversible, firms cannot disinvest even when the marginal profitability of capital is low. Anticipating that this constraint may bind in the future, firms apply a higher user cost of capital to current investment decisions. Our analysis of the expected long-run capital stock demonstrates that both the "feared event" (inability to disinvest, summarized by the hangover effect) and the firm's reaction to it (a high user cost, summarized by the user-cost effect) are important features of capital

16 This does not imply that the firm does not choose its capital stock optimally, but that it chooses its optimal capital stock conditional on the irreversibility constraint. 
accumulation. Relative to the (Jorgensonian) case of reversible investment, the inability to rid itself of capital increases the firm's capital stock during "bad" times, and decreases it during "good" times. In the long run, either effect can dominate, so that the expected capital stock may be higher or lower under irreversibility than under reversibility.

Uncertainty does not ease the ambiguity regarding the longrun effect of irreversibility, but rather deepens it. Higher uncertainty strengthens both the hangover and user-cost effects, but which effect becomes relatively stronger again depends on characteristics of the firm and its environment. Higher uncertainty increases the level of the expected long-run capital stock under both irreversibility and reversibility (for $0<\theta<1$ ), but in which case the expected capital stock increases more is ambiguous.

While in general it is not possible to determine whether irreversibility increases or decreases the expected long-run capital stock of a firm, other characteristics of the firm provide some clues. We find that a high growth rate of demand decreases the expected long-run capital stock under irreversibility relative to that under reversibility. On the other hand, high interest rates, a low capital share in production, and a low elasticity of demand have the opposite effect -- tending to increase the expected longrun capital stock with irreversibility relative to that under reversibility. Together, these results suggest that a firm's long-run expected capital stock is most likely to be decreased by the presence of irreversibility in an environment with high demand 
growth, low interest rates, a high capital share, and highly elastic demand.

These results are obtained in a specific framework, using particular functional forms and assumptions. They may not be general, in that the introduction of equilibrium considerations, different specifications for uncertainty, operating profits, or investment costs, or other generalizations may affect our findings. Simple models such as the one we use, however, have been employed to illustrate the importance of irreversibility and uncertainty for investment and hiring dynamics. Our results demonstrate that even in such models, where changes in the user cost due to irreversibility and uncertainty change investment and employment flows in sometimes dramatic fashion, these effects may be dampened or even reversed when translated into the expected long-run stock. Thus, the effect on the expected longrun stock of capital or labor may be smaller or even of the opposite sign than the effect of irreversibility and uncertainty on the user cost would indicate. 
Assume that the firm is risk-neutral and discounts future cash flows at the constant rate $r>0$, where $r>\mu .{ }^{17}$ The fundamental value of the firm is

$V\left(K_{t}, X_{t}\right) \equiv \max _{\left\{l_{t+t}\right\}} E_{t}\left\{\int_{0}^{\infty} e^{-r t}\left[\pi\left(K_{t+s}, X_{t+s}\right) d s-b d K_{t+s}\right]\right\} \cdot$ (A.1)

Since $K_{t}$ is not differentiable, the last term in equation (A.1) is to be interpreted as a Stieltjes integral.

The value function in equation (A.1) is homogeneous of degree one in $K_{t}$ and $X_{t}$. Therefore, the marginal valuation of capital, $V_{K}\left(K_{t}, X\right)$, is homogeneous of degree 0 in $K_{t}$ and $X_{t}$ and can be written as a function of $y_{t} \equiv X_{t} / K_{t}$. Define

$$
q\left(y_{t}\right) \equiv V_{K}\left(K_{t}, X_{t}\right)
$$

as the marginal valuation of capital. Optimality requires

$$
r q(y)=h y^{\gamma}+\frac{1}{d t} E\{d q\}
$$

${ }^{17}$ Under optimal policy the marginal revenue product of capital, $h X^{\gamma} K^{-\gamma}$, satisfies $h X^{\gamma} K^{-\gamma} \leq c$ where $c$ is the user cost of capital. Recalling that $0<\gamma<1$, so that $(\gamma$ 1) $\gamma<0$, this inequality is equivalent to $h^{(\gamma-1) / \gamma} X^{\gamma-1} K^{1-\gamma} \geq c^{(\gamma-1) / \gamma}$. Multiplying this inequality by $\left(h^{1 / \gamma /(1-\gamma)) X}\right.$ and recalling that $\pi(K, X)=\left(h /(1-\gamma) X^{\gamma} K^{1-\gamma}\right.$, we obtain $\pi(X, K) \geq\left(h^{1 / r} /(1-\gamma)\right) c^{(r-1) / r} X$. Since the expected growth rate of $X$ is $\mu$, the operating profit of the firm is bounded below by a process with expected growth rate equal to $\mu$ Therefore, in order for the expected present value of operating profits, $E_{t}\left\{\int_{0}^{\infty} e^{-r s} \pi\left(K_{t+s}, X_{t+s}\right) d s\right\}$, to be finite we must assume that $\mu<r$. 
where the left hand side is the required return on the marginal unit of capital, and the right hand side is the expected return on the marginal unit of capital, which consists of the marginal revenue product, $h y^{\gamma}$, and the expected capital gain $(1 / d t) E\{d q\}$.

When the marginal valuation of capital, $q(y)$, is less than the purchase price of capital $b$, it is optimal not to purchase capital. It is optimal to purchase capital only if $q(y)$ reaches the boundary $b$. The value of $y$ at this boundary, $y_{U}$, is given by the smoothpasting condition ${ }^{18}$

$$
q\left(y_{U}\right)=b \text {. }
$$

In addition, $q(y)$ and $y_{U}$ must satisfy the high-contact condition

$$
q^{\prime}\left(y_{U}\right)=0
$$

To calculate the expected change in $q$ apply Ito's Lemma to $q(y)$ and use the high-contact condition in equation (A.5) to obtain $^{19}$

$$
\frac{1}{d t} E\{d q\}=\mu y q^{\prime}(y)+\frac{1}{2} \sigma^{2} y^{2} q^{\prime \prime}(y)
$$

\footnotetext{
${ }^{18}$ See Dumas (1991) for a clear presentation of the smooth-pasting and high-contact conditions used below.

${ }^{19}$ Note that $\frac{1}{d t} E\{d q\}=\mu v q^{\prime}(y)-y q^{\prime}(y) \frac{d K}{K}+\frac{1}{2} \sigma^{2} y^{2} q^{\prime \prime}(y)$. When $y \neq y_{U}$, $d K=0$; when $y=y_{1} q^{\prime}(y)=0$ by the high-contact condition. Therefore, $q^{\prime}(y) d K=0$ always, and we obtdin equation (A.6).
} 
Substituting equation (A.6) into the optimality condition in equation (A.3) yields a second-order differential equation that $q(y)$ must satisfy

$$
r q(y)=h y^{\gamma}+\mu y q^{\prime}(y)+\frac{1}{2} \sigma^{2} y^{2} q^{\prime \prime}(y)
$$

The solution to the differential equation (A.7) is ${ }^{20}$

$$
q(y)=A y^{r}+J y^{\alpha_{p}}, \text { where } A \equiv \frac{h}{\rho(\gamma)}>0
$$

and $\alpha_{P}$ is the positive root of the quadratic equation in equation (5b), and $J$ is a constant that will be determined by the boundary conditions. Using equation (A.8) in the boundary conditions, equations (A.4, A.5), we obtain

$$
h y_{U}^{\gamma}=c \equiv\left(1-\frac{\gamma}{\alpha_{N}}\right) r b
$$

and

$$
q(y)=A y^{\gamma}-\frac{\gamma}{\alpha_{P}-\gamma} b\left(\frac{y}{y_{U}}\right)^{\alpha_{p}}
$$

\footnotetext{
${ }^{20}$ The general solution to the differential equation (A.7) contains a third term $L y^{\alpha_{N}}$, where $L$ is a constant. Note that as $y$ approaches zero, this term approaches infinity because $\alpha_{N}<0$. However, the marginal valuation of capital remains finite as $y \equiv X / K$ approaches zero. Therefore the constant $L$ must equal zero.
} 
Appendix B. Properties of the negative root of $\rho(\eta)=0$ in equation (5b)

Recall that $\rho(\eta) \equiv-\frac{1}{2} \sigma^{2} \eta^{2}-\left(\mu-\frac{1}{2} \sigma^{2}\right) \eta+r=0$. Note that $\rho(\eta)$ is strictly concave, $\rho(0)=r>0$, and $\rho(1)=r-\mu>0$ so that $\rho(\gamma)>0$. Also, note that $\rho(\eta)=0$ has two distinct roots, $\alpha_{P}>0$ and $\alpha_{N}<0$, which satisfy $\alpha_{N}<0<\gamma<1<\alpha_{P}$. The concavity of $\rho(\eta)$ implies that

$$
\rho^{\prime}\left(\alpha_{N}\right)=-\sigma^{2} \alpha_{N}-\mu+\frac{1}{2} \sigma^{2}>0
$$

Totally differentiating $\rho(\eta)$ with respect to $\eta, \mu, \sigma^{2}$, and $r$, and evaluating the derivatives at $\eta=\alpha_{N}$ yields

$\rho^{\prime}\left(\alpha_{N}\right) d \alpha_{N}-\alpha_{N} d \mu+\frac{1}{2} \alpha_{N}\left(1-\alpha_{N}\right) d \sigma^{2}+d r=0$

Equation (B.2) implies

$$
\begin{aligned}
& \frac{d \alpha_{N}}{d \mu}=\frac{\alpha_{N}}{\rho^{\prime}\left(\alpha_{N}\right)}<0 \\
& \frac{d \alpha_{N}}{d r}=-\frac{1}{\rho^{\prime}\left(\alpha_{N}\right)}<0
\end{aligned}
$$


Now we calculate the effect on the root $\alpha_{N}$ of a $M(\theta)$-preserving increase in $\sigma^{2}$. Observe from equation (16) that for a $M(\theta)$ preserving change

$$
d \mu=\frac{1}{2}(1-\theta) d \sigma^{2}
$$

Substituting equation (B.5) into equation (B.2) yields

$$
\left.\frac{d \alpha_{N}}{d \sigma^{2}}\right|_{M(\theta)=M_{0}}=\frac{1}{2} \frac{\alpha_{N}\left(\alpha_{N}-\theta\right)}{\rho^{\prime}\left(\alpha_{N}\right)}
$$

Note from equation (B.6) that if $\theta>0$, then $\left.\frac{d \alpha_{N}}{d \sigma^{2}}\right|_{M(\theta)=M_{0}}>0$.

Lemma B.1: If $\mu>(1 / 2) \sigma^{2}$, then $\alpha_{N}<-1$.

Proof: Observe that $\rho(0)=r>0$ and $\rho(-1) \equiv \mu+r-\sigma^{2}>2 \mu-\sigma^{2} \geq 0$. Therefore, $\alpha_{N}$ is not in the interval $[-1,0]$.

q.e.d. 


\section{Appendix C: Proof of Proposition 1}

\section{Lemma C.1:}

If $\omega(\gamma) \equiv \ln \left(1-\frac{\gamma}{\alpha_{N}}\right)^{-1 / \gamma}=-\frac{1}{\gamma} \ln \left(1-\frac{\gamma}{\alpha_{N}}\right)<0$, then $\omega(0)=\frac{1}{\alpha_{N}}$.

Proof: Apply L'Hopital's Rule to the ratio $N(\eta) / D(\eta)$ where $N(\gamma) \equiv-\ln \left(1-\frac{\gamma}{\alpha_{N}}\right)$ and $D(\gamma) \equiv \gamma$. Observe that $N^{\prime}(\gamma)=\frac{1 / \alpha_{N}}{1-\frac{\gamma}{\alpha_{N}}}$ so that $N^{\prime}(0)=1 / \alpha_{N}$. Also $D^{\prime}(0)=1$.

Therefore, $\omega(0)=N^{\prime}(0) / D^{\prime}(0)=1 / \alpha_{N}$

q.e.d.

\section{Lemma C.2}

If $\omega(\gamma) \equiv \ln \left(1-\frac{\gamma}{\alpha_{N}}\right)^{-1 / \gamma}=-\frac{1}{\gamma} \ln \left(1-\frac{\gamma}{\alpha_{N}}\right)$, then $\omega^{\prime}(\gamma) \geq 0$ with strict inequality for $\gamma>0$.

Proof:

$$
\omega^{\prime}(\gamma)=\frac{1}{\gamma^{2}} \ln \left(1-\frac{\gamma}{\alpha_{N}}\right)+\frac{1}{\gamma} \frac{1 / \alpha_{N}}{1-\frac{\gamma}{\alpha_{N}}}=\frac{1}{\gamma^{2}}\left[\ln \left(1-\frac{\gamma}{\alpha_{N}}\right)+\frac{\gamma / \alpha_{N}}{1-\frac{\gamma}{\alpha_{N}}}\right] .
$$

Therefore, $\omega^{\prime}(\gamma)=\frac{1}{\gamma^{2}} \gamma\left(\frac{-\gamma}{\alpha_{N}}\right)$, where $\gamma(z) \equiv \ln (1+z)-\frac{z}{1+z}$.

Note that $v(0)=0$ and $v(z)=\frac{z}{(1+z)^{2}}$ so that $v(z)>0$ for $z>0$.

Therefore, $\omega^{\prime}(\eta)>0$.

q.e.d. 
Proof of Proposition 1: It follows from the definition of $C$ in equation (15b) and the assumption that $\gamma>0$ that $C<1$. Lemma C. 2 and the assumption that $y>0$ imply that $C=e^{\Delta(\gamma)}>e^{a(0)}$. Using the expression for $\omega(0)$ in Lemma $C .1$ yields $C>e^{1 / \alpha_{N}}$. Lemma B. 1 implies that $e^{1 / \alpha_{N}}>e^{-1}=0.367879$.

q.e.d. 
Appendix D: The Effect of Demand Growth, the Interest

Rate, the Capital Share, and the Price Elasticity of Demand on the Expected Long-run Capital Stock

Proof of Proposition 7: Differentiating the expression for $H(\infty)$ in Proposition 2 with respect to $\mu$ yields

$$
\frac{d H(\infty)}{d \mu}=-\frac{\sigma^{2}}{2 \mu^{2}}<0
$$

Turning now to $\frac{d C}{d \mu}$, note that $\mu$ enters the expression for $C$ in equation (15b) only through its effect on the root $\alpha_{N}$.

Differentiating equation (15b) with respect to $\alpha_{N}$ yields

$$
\frac{d C}{d \alpha_{N}}=-\frac{1}{\gamma} \frac{C}{1-\frac{\gamma}{\alpha_{N}}} \frac{\gamma}{\alpha_{N}^{2}}=\frac{C}{\alpha_{N}\left(\gamma-\alpha_{N}\right)}<0
$$

Differentiating equation (15b) with respect to $\mu$, and using equations (D.2) and (B.3) yields

$$
\frac{d C}{d \mu}=\frac{d C}{d \alpha_{N}} \frac{d \alpha_{N}}{d \mu}=\frac{C}{\left(\gamma-\alpha_{N}\right) \rho^{\prime}\left(\alpha_{N}\right)}>0
$$

\section{q.e.d.}

Proof of Corollary to Proposition 7: Using equations (D.1) and (D.3) and the fact that $H(\infty)=1+\sigma^{2} /(2 \mu)$ yields

$$
\frac{d \kappa(\infty)}{d \mu}=H(\infty) \frac{d C}{d \mu}+C \frac{d H(\infty)}{d \mu}=\frac{\left(1+\frac{\sigma^{2}}{2 \mu}\right) C}{\left(\gamma-\alpha_{N}\right) \rho^{\prime}\left(\alpha_{N}\right)}-C \frac{\sigma^{2}}{2 \mu^{2}}
$$

which can be rearranged as 
$\frac{d \kappa(\infty)}{d \mu}=\frac{C}{\mu\left(\gamma-\alpha_{N}\right) \rho^{\prime}\left(\alpha_{N}\right)}\left[\mu+\frac{1}{2} \sigma^{2}-\frac{\sigma^{2}}{2 \mu}\left(\gamma-\alpha_{N}\right) \rho^{\prime}\left(\alpha_{N}\right)\right]$

Define $G \equiv \frac{\sigma^{2}}{2 \mu}>0$ and rewrite equation (D.5) as

$\frac{d \kappa(\infty)}{d \mu}=\frac{C}{\left(\gamma-\alpha_{N}\right) \rho^{\prime}\left(\alpha_{N}\right)}\left[1+G+G \alpha_{N} \frac{\rho^{\prime}\left(\alpha_{N}\right)}{\mu}-G \gamma \frac{\rho^{\prime}\left(\alpha_{N}\right)}{\mu}\right]$

Recall that $\rho^{\prime}\left(\alpha_{N}\right)>0$. Thus, to show that $d \kappa(\infty) / d \mu<0$ it suffices to show that $1+G+G \alpha_{N} \rho^{\prime}\left(\alpha_{N}\right) / \mu<0$.

Evaluate the expression for $\rho(\eta)$ in equation (5b) at $\eta=-G^{-1}$ to obtain

$\rho\left(-G^{-1}\right) \equiv-\frac{1}{2} \sigma^{2} G^{-2}+\left(\mu-\frac{1}{2} \sigma^{2}\right) G^{-1}+r=r-\mu>0$ (D.7)

where the inequality follows from our assumption that $r>\mu$. The concavity of $\rho(\eta)$ and the fact that $\rho\left(-G^{-1}\right)>0$ imply $\alpha_{N}<-G^{-1}$, which implies that

$$
-\alpha_{N} G>1 .
$$

Observe from equations (B.1) and (D.8) that

$$
\frac{\rho^{\prime}\left(\alpha_{N}\right)}{\mu}=-2 G \alpha_{N}-1+G>1+G
$$

It follows from equations (D.8) and (D.9) that

$$
1+G+G \alpha_{N} \frac{\rho^{\prime}\left(\alpha_{N}\right)}{\mu}<0
$$


As discussed below equation (D.6), the condition in equation

(D. 10) is sufficient to prove that $d \kappa(\infty) / d \mu<0$.

q.e.d.

Proof of Proposition 8: Notice from the definition of $H(t)$ in equation (15c) that it is independent of $r$ for all $t$. Also, from equation (15b) $r$ affects $C$ only through its effect on the root, $\alpha_{N}$. Thus, using equations (B.4) and (D.2) we obtain

$$
\begin{aligned}
\frac{d \kappa(t)}{d r} & =\frac{d C}{d r} H(t)=\frac{d C}{d \alpha_{N}} \frac{d \alpha_{N}}{d r} H(t) \\
& =\frac{-\kappa(t)}{\alpha_{N}\left(\gamma-\alpha_{N}\right) \rho^{\prime}\left(\alpha_{N}\right)}>0
\end{aligned}
$$

q.e.d.

Proof of Proposition 9: Notice from the definition of $H(t)$ in equation (15c) that it is independent of $\gamma$ for all $t$. Also note that $\rho(\eta)$ is independent of $\gamma$ so that $\alpha_{N}$ is independent of $\gamma$. Thus, using the definition of $C$ in equation (15b) and differentiating with respect to $\gamma$, we obtain

$$
\frac{d \kappa(t)}{d \gamma}=\frac{d C}{d \gamma} H(t)=\frac{\kappa(t)}{\gamma^{2}}\left[\ln \left(1-\frac{\gamma}{\alpha_{N}}\right)-\frac{\gamma}{\gamma-\alpha_{N}}\right]
$$

The expression in equation (D.12) will be positive if and only if $D\left(\alpha_{N}\right)>0$ where 


$$
D\left(\alpha_{N}\right) \equiv \ln \left(1-\frac{\gamma}{\alpha_{N}}\right)-\frac{\gamma}{\gamma-\alpha_{N}}
$$

Observe that $D^{\prime}\left(\alpha_{N}\right)=-\frac{\gamma^{2}}{\alpha_{N}\left(\alpha_{N}-\gamma\right)^{2}}>0$ which implies that

$$
D\left(\alpha_{N}\right)>D(-\infty) \equiv \ln (1)-0=0
$$

q.e.d. 


\section{References}

Abel, Andrew B., "Optimal Investment Under Uncertainty," American Economic Review, 73, 1 (March 1983), 228-233.

Abel, Andrew B. and Janice C. Eberly, "An Exact Solution for the Investment and Market Value of a Firm Facing Uncertainty, Adjustment Costs, and Irreversibility," National Bureau of Economic Research Working Paper No. \#4412, July 1993.

Abel, Andrew B. and Janice C. Eberly, "Optimal Investment with Costly Reversibility," National Bureau of Economic Research Working Paper No. \#5091, April 1995.

Bentolila, Samuel and Giuseppe Bertola, "Firing Costs and Labor Demand: How Bad is Eurosclerosis?" Review of Economic Studies, 57 (1990), 381-402.

Bertola, Giuseppe, "Adjustment Costs and Dynamic Factor Demands: Investment and Employment Under Uncertainty," Ph.D. Dissertation, Cambridge, MA: M.I.T., 1988.

Bertola, Giuseppe, "Labor Turnover Costs and Average Labor Demand," Journal of Labor Economics, 10(4), 1992, pp. 389-411.

Bertola, Giuseppe and Ricardo Caballero, "Irreversibility and Aggregate Investment," Review of Economic Studies, 61 (1994), 223-246.

Caballero, Ricardo J., "On the Sign of Investment-Uncertainty Relationship," American Economic Review, 81, 1 (March 1991), 279-288.

Dixit, Avinash, "Entry and Exit Decisions under Uncertainty," Journal of Political Economy, 97, 3 (June 1989), 620-638.

Dixit, Avinash, "Irreversible Investment with Price Ceilings," Journal of Political Economy, 99, 3 (June 1991), 541-557. 
Dixit, Avinash, "Investment and Hysteresis," Journal of Economic Perspectives, 6(1) (1992), 107-132.

Dixit, Avinash and Robert S. Pindyck, Investment Under Uncertainty, Princeton, NJ: Princeton University Press, 1994.

Dumas, Bernard, "Super Contact and Related Optimality Conditions," Journal of Economic Dynamics and Control, 15, 4 (October 1991), 675-685.

Goldman, M. Barry, Howard B. Sosin and Mary Ann Gatto, "Path Dependent Options: "Buy at the Low, Sell at the High," Journal of Finance, 34, 5 (December 1979), 1111-1127.

Harrison, J. Michael, Brownian Motion and Stochastic Flow Systems, Malabar, FL: Krieger Publishing Company, 1985.

Hartman, Richard, "The Effects of Price and Cost Uncertainty on Investment, Journal of Economic Theory, 5, (October 1972), 258266.

Jorgenson, Dale W., "Capital Theory and Investment Behavior," American Economic Review Papers and Proceedings, 53 (May 1963), 247-259.

Pindyck, Robert S., "Irreversible Investment, Capacity Choice, and the Value of the Firm," American Economic Review, 78, 5 (December 1988), 969-985.

Pindyck, Robert S., "A Note on Competitive Investment Under Uncertainty," American Economic Review, 83, 1 (March 1993), 273-277. 